\title{
A Coupled Level Set and Particle-In-Cell Method for Penetration Problems
}

\author{
Zhang $\mathrm{Li}^{1,2}$, Zhang Tao ${ }^{2, *}$, Wu Kaiteng ${ }^{2}$ and Ning Jianguo ${ }^{1}$ \\ ${ }^{1}$ State Key Laboratory of Explosion Science and Technology, Beijing Institute of Technology, Beijing 100081, China; \\ ${ }^{2}$ Key Laboratory of Numerical Simulation of Sichuan Province, Neijiang Normal University, Neijiang 641110, China
}

\begin{abstract}
A coupled level set and Particle-In-Cell method is presented to treat the penetration problems. The coupled method has the advantages of level set method and Particle-In-Cell method, and the basic idea is that the important computation region such as the large deformation region where more particles are added for calculation, while the continuous transport method is used in the other regions. The steel projectile penetration into concert target is simulated by using the above-mentioned coupled method. The numerical results reveal that the adopted coupled method can effectively track the deformation in the process of penetration and also insure efficiency and precision.
\end{abstract}

Keywords: Level set method, particle-in-cell, penetration.

\section{INTRODUCTION}

Penetration refers to a projectile, fragment or shaped charge jet with a certain speed penetrating or perforating the target and causing the damage effect with its own kinetic energy [1-3]. The penetration issues under impact load are a complex nonlinear dynamic response process, which involves not only geometric nonlinearity caused by the large structural displacement, but also involves the physical nonlinearity caused by the large material deformation. In addition, it involves complex kinematic nonlinearity and contact and friction problems. For now, there are basically two methods such as Lagrange method and Eulerian method for numerical simulation penetration problems. They have their own advantages and limitations, and Lagrange method has become very mature. Though the Eulerian method has its advantage in treating large deformation problems, when the studied system includes multi-materials, how well the method can deal with the mixed cells becomes very important. How to determine the multi-material interface in the mixed cells, and how to calculate the physical quantities in the mixed cells to the neighboring cells are difficult with the Eulerian method. From the 1950 s to the 1960 s, the simulation of free surface and material interface had been substantially developed. The researchers of Los Alamos national laboratory in America proposed and developed the Cell-type method [4-8], with very effective use. But the method must calculate and store not only the information of cells, but also the parameters of particles. In 1988, Osher and Sethian published the famous level set method $[9,10]$, which contributed significantly to the simulation of interface tracking [11]. At present, level set method is widely used because it is easy and effective. However, it has disadvantages in treating fracture of material due to the non-conservation of volume. In order to balance the advantages of both Particle-In-Cell and level set method, a few coupled methods $[12,13]$ are developed by many pioneering researchers.

In this paper, a coupled level set and Particle-In-Cell method is proposed for treating multi-material interface. This method is used in the important computational region such as the large deformation region, fracture region and material interface where more particles are added for calculation, while the continuous transport method is used in the other regions. The method ensures the computational accuracy of the important region, saving the computational time, and improving the computational efficiency. The steel projectile penetration into concert target is simulated by using the above-mentioned coupled method.

\section{GOVERNING EQUATIONS}

In two-dimensional axis-symmetric coordinates, the mass, momentum and energy conservation equations are as follows.

$$
\begin{aligned}
& \frac{\partial \rho}{\partial t}+u_{z} \frac{\partial \rho}{\partial z}+u_{r} \frac{\partial \rho}{\partial r}+\rho\left(\frac{\partial u_{z}}{\partial z}+\frac{\partial r u_{r}}{r \partial r}\right)=0 \\
& \rho\left(\frac{\partial u_{z}}{\partial t}+u_{z} \frac{\partial u_{z}}{\partial z}+u_{r} \frac{\partial u_{z}}{\partial r}\right)=-\frac{\partial P}{\partial z}+\frac{\partial S_{z z}}{\partial z}+\frac{\partial\left(r S_{r z}\right)}{r \partial r} \\
& \rho\left(\frac{\partial u_{r}}{\partial t}+u_{z} \frac{\partial u_{r}}{\partial z}+u_{r} \frac{\partial u_{r}}{\partial r}\right)=-\frac{\partial P}{\partial r}+\frac{\partial S_{r z}}{\partial z}+\frac{\partial\left(r S_{r r}\right)}{r \partial r}+\frac{\left(S_{r r}+S_{z z}\right)}{r} \\
& \rho\left(\frac{\partial e}{\partial t}+u_{z} \frac{\partial e}{\partial z}+u_{r} \frac{\partial e}{\partial r}\right)=-P\left(\frac{\partial\left(r u_{r}\right)}{r \partial r}+\frac{\partial u_{z}}{\partial z}\right)+S_{z z} \frac{\partial u_{z}}{\partial z} \\
& +S_{r r} \frac{\partial u_{r}}{\partial r}+S_{r z}\left(\frac{\partial u_{r}}{\partial r}+\frac{\partial u_{r}}{\partial z}\right)-\frac{u_{r}\left(S_{r r}+S_{z z}\right)}{r}
\end{aligned}
$$

where, $t$ is time, $\rho$ is the density, $e$ is the specific internal energy, $r$ and $z$ are the radial and axial coordinates of the 
cylindrical coordinate system, respectively, $u_{r}$ and $u_{z}$ are the radial and axial components of velocity, $S_{r r}, S_{z z}, S_{r z}$ are components of stress deviator tensor.

\section{COUPLED LEVEL SET AND PARTICLE-IN-CELL METHOD}

\subsection{Particle in Cell Method}

This method takes the continuum as composed of particles which have mass. It achieves the numerical simulation of the flow field and interface display by computing and tracking the particles.

Assuming that a cell has $N_{\max }$ particles, the coordinates $\left(r_{n}, z_{n}\right)$ of the $n$th $\left(0 \leq n \leq N_{\max }\right)$ particle are obtained using the following formula:

$$
\begin{aligned}
& r_{n}=j+\left(\frac{1}{2}+a\right) \cdot \frac{\Delta r}{\sqrt{N_{\text {max }}}} a=n \% \sqrt{N_{\text {max }}} \\
& z_{n}=i+\left(\frac{1}{2}+b\right) \cdot \frac{\Delta z}{\sqrt{N_{\text {max }}}} a=n \sqrt{N_{\text {max }}}
\end{aligned}
$$

where $i$ and $j$ are cell coordinates, $\Delta z$ and $\Delta r$ are spatial steps, $a, b, c$, are pure cell, pure cell and mixed cell Fig. (1).

The velocities $u_{z}$ and $u_{r}$ of particle $p_{n}$ are calculated from adjacent four cells by area-weighted method as shown in Fig. (2). The formula is as follows:

$$
\begin{aligned}
& u_{z}=\frac{1}{\Delta r \Delta z}\left(A_{1}\left[u_{z}\right]_{i+1, j+1}+A_{2}\left[u_{z}\right]_{i, j+1}+A_{3}\left[u_{z}\right]_{i, j}+A_{4}\left[u_{z}\right]_{i+1, j}\right) \\
& u_{r}=\frac{1}{\Delta r \Delta z}\left(A_{1}\left[u_{r}\right]_{i+1, j+1}+A_{2}\left[u_{r}\right]_{i, j+1}+A_{3}\left[u_{r}\right]_{i, j}+A_{4}\left[u_{r}\right]_{i+1, j}\right)
\end{aligned}
$$

where

$$
\begin{aligned}
& A_{1}=\left[z_{n}-\left(i+\frac{1}{2}\right) \Delta z\right]\left[r_{n}-\left(j+\frac{1}{2}\right) \Delta r\right] \\
& A_{2}=\left[z_{n}-\left(i+\frac{1}{2}\right) \Delta z\right]\left[\left(j+\frac{3}{2}\right) \Delta r-r_{n}\right] \\
& A_{3}=\left[\left(i+\frac{3}{2}\right) \Delta z-z_{n}\right]\left[\left(j+\frac{3}{2}\right) \Delta r-r_{n}\right] \\
& A_{4}=\left[\left(i+\frac{3}{2}\right) \Delta z-z_{k}\right]\left[r_{n}-\left(j+\frac{1}{2}\right) \Delta r\right]
\end{aligned}
$$

If the particle is not located on the bottom left corner, the corresponding adjacent four cells are adopted, and the calculation procedure is similar.

The new position $\left(r_{n}^{k+1}, z_{n}^{k+1}\right)$ of the particle $p_{n}$ at the time step $n+1$ is calculated as follows:

$$
\begin{aligned}
& r_{n}^{k+1}=r_{n}^{k}+u_{r} \cdot \Delta t \\
& z_{n}^{k+1}=z_{n}^{k}+u_{z} \cdot \Delta t
\end{aligned}
$$

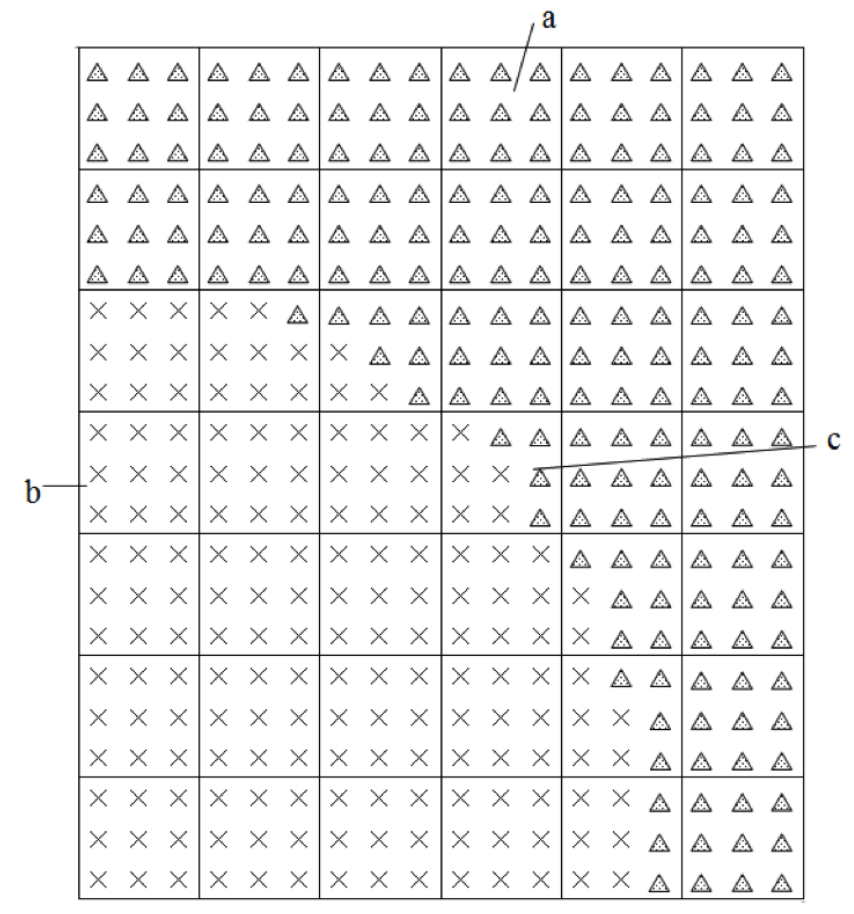

Fig. (1). Particle configuration in Particle-In- Cell Method.

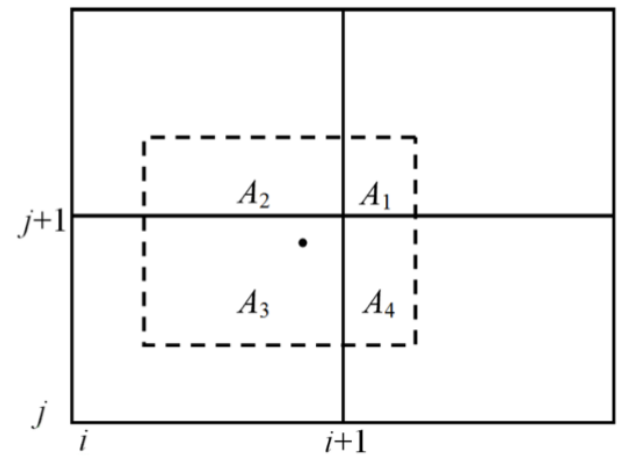

Fig. (2). Schematic representation of the area-weighted method.

If a particle $\left(r_{n}^{k+1}, z_{n}^{k+1}\right)$ moves across the boundary of the $(i, j)$ th cell, its mass, momentum and energy are subtracted from the donor cell and added to the acceptor cell. Thus a cycle of transport calculation in the Eulerian step is completed.

\subsection{Ghost and Grid Method}

In 1988, Osher proposed the level set method, which is an effective method, is used to describe the location interface. The basic idea is that construct function $\varphi(\vec{x}, t)$ at any moment, the moving interface is exactly the zero isosurface, that is

$\Gamma(t)=\{\vec{x} \in \Omega: \varphi(\vec{x}, t)=0\}$

For example, two-dimensional, level set equation

$\varphi_{t}+u(\vec{x}) \varphi_{x}+v(\vec{x}) \varphi_{y}=0$

Where $u$ and $v$ are the component in the $\vec{x}=(x, y)$ direction of the velocity vector $\vec{V}$. 
At any given moment, if $\varphi(\vec{x}, \vec{y}, t)$ satisfies $\varphi(\vec{x}, \vec{y}, t)=0$, then $\Gamma(t)=\{(\vec{x}, \vec{y}) \in \Omega: \varphi(\vec{x}, \vec{y}, t)=0\}$ is the interface. But the key question is solving physical quantity in the vicinity of the interface. Because solve internal physical quantity must use external physical quantity, however, internal physical quantity and external physical quantity are located in both sides of the interface. Assuming that the function value is known in $k$ th time horizon, it can't use the function value in $k$ th time horizon for the function value in $(k+1)$ th time horizon.

In order to solve interface discontinuity problems, Osher and Glimm first used the ghost grid method [14, 15], and it usually uses "isobaric fix $[16,17]$ " techniques to reduce the "overheating" phenomenon. As a one dimensional example, suppose the interface of fluid $A$ and $B$ is the between $i$ th grid points and $(i+1)$ th grid points Fig. (3), that is level set function exists zero value between $i$ th grid points and $(i+1)$ th grid points. We define

$P_{i+1}^{A}=P_{i+1}^{B}, \quad V_{i+1}^{A}=V_{i+1}^{B}, \quad S_{i+1}^{A}=g S_{i-1}^{A}$

where $g$ is extrapolation function.

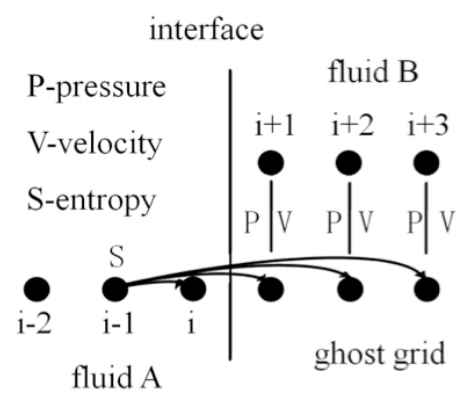

Fig. (3). Ghost method with isobaric fix technology.

\subsection{The Coupled Method}

In order to assure the accuracy of contact region, without increasing the scale of computation, this article proposes a coupled level set and Particle-In-Cell method. The basic idea is that for the important region, a large number of particles are added to ensure the computational precision, while the other regions still use the continuous transport method. Based on the characteristics of cells, the coupled method can be divided into four cases

(1) For the cell containing particles absolutely, including the pure cell contains one kind of particle and the mixed cell which contains two kinds of particle, the Particle-In-Cell method in mentioned previously is adopted.

For the donor cell

$M_{i j}^{n+1}=M_{i j}^{n}-\sum_{k} m_{k},(0<k \leq N)$

For the acceptor cell

$M_{i j}^{n+1}=M_{i j}^{n}+\sum_{k} m_{k},(0<k \leq N)$

(2) For the pure cell not containing particle, the continuous transport method is adopted.
For the donor cell

$M_{i j}^{n+1}=M_{i j}^{n}-C$

For the acceptor cell

$M_{i j}^{n+1}=M_{i j}^{n}+C$

(3) For the half-cell containing no particle at the boundary of the object, the Ghost method with isobaric fix technology is adopted.

(4) With the calculation going on, some cell will contain discrete and continuous medium at the same time. Then, the coupled method adopts discrete transport for particle and Ghost method with isobaric fix technology for continuous medium. Especially, the position of the Ghost interface in this cell is determined by the mass friction when the discrete particle and continuous medium is treated as two materials.

For the donor cell

$\left\{\begin{array}{c}M_{i j}^{n+1}=M_{i j}^{n}-\sum_{k} m_{k},(0<k \leq N) \\ M_{i j}^{n+1}=M_{i j}^{n}-C\end{array}\right.$

For the acceptor cell

$\left\{\begin{array}{c}M_{i j}^{n+1}=M_{i j}^{n}+\sum_{k} m_{k},(0<k \leq N) \\ M_{i j}^{n+1}=M_{i j}^{n}+C\end{array}\right.$

In equations (12)-(17), $M$ represents the total mass, momentum and energy of the cell, $m$ is the mass, momentum and energy of the particle, $N$ is the number of particle transported or accepted, $C$ is the transport velocity, mass and energy calculated by the continuous transport method.

\section{NUMERICAL SIMULATION OF POINTED PRO- JECTILE PENETRATION INTO STEEL TARGET}

\subsection{Computational Model}

The computational model is selected as shown in Fig. (4). Due to the two-dimensional axis-symmetric character, the quarter model is selected for the computation. For the bottom boundary, the axis--symmetric boundary condition is adopted. For the other three boundaries, the continuous boundary condition is used. The projectile length is $36 \mathrm{~mm}$ $\left(L_{1}+L_{2}\right)$, with a diameter of $12 \mathrm{~mm}$. The target thickness is $6 \mathrm{~mm}$, with both length and width of $120 \mathrm{~mm}$. The impact velocity of the projectile is $302.5 \mathrm{~m} / \mathrm{s}$. The steel projectile model is rigid material, and the parameters of steel projectile are shown in Table 1. The Johnson-Cook model is used for concert target, and the material parameters of concrete target is shown in Table 2 . The size of the computational region is $120 \mathrm{~mm} \times 120 \mathrm{~mm}$, and 1080 particle are added to the contact region of the projectile and target.

\subsection{Numerical Results and Analysis}

Fig. (5) present penetration process at different time and different perspectives. Fig. (6) described the particle region, which clearly reveals the deformation of the target. The 
Table 1. Material constants for steel projectile.

\begin{tabular}{|c|c|c|c|c|}
\hline Material & $\rho\left(\mathbf{k g} / \mathbf{m}^{-3}\right)$ & $\boldsymbol{E} / \mathbf{G P a}$ & $\sigma_{\mathrm{s}} / \mathbf{G P a}$ & $v$ \\
\hline \hline $\mathrm{T}-250$ & $7.83 \times 10^{3}$ & 208 & 1.825 & 0.35 \\
\hline
\end{tabular}

Table 2. Material constants for target.

\begin{tabular}{|c|c|c|c|c|}
\hline Parameters & $\rho\left(\mathrm{kg} / \mathrm{m}^{-3}\right)$ & $E / G P a$ & $\sigma_{\mathrm{s}} / \mathbf{G P a}$ & $v$ \\
\hline Value & $7.83 \times 10^{3}$ & 2 & 0.35 & 0.31 \\
\hline Parameters & $A / \mathbf{G P a}$ & $B / \mathbf{G P a}$ & G/ GPa & $n$ \\
\hline Value & 0.079 & 0.051 & 0.014 & 0.26 \\
\hline Parameters & $m$ & $T_{m}$ & C & $S_{1}$ \\
\hline Value & 1.03 & 1793 & 0.4523 & 1.49 \\
\hline Parameters & $c$ & $\gamma_{0}$ & $\alpha$ & $C_{v}$ \\
\hline Value & 0.025 & 2.18 & 0.46 & $4.77 \mathrm{e}-6$ \\
\hline Parameters & $D_{1}$ & $S_{R}$ & $V_{0}$ & $E_{0}$ \\
\hline Value & 0.8 & $1 e-6$ & 1 & 0 \\
\hline
\end{tabular}

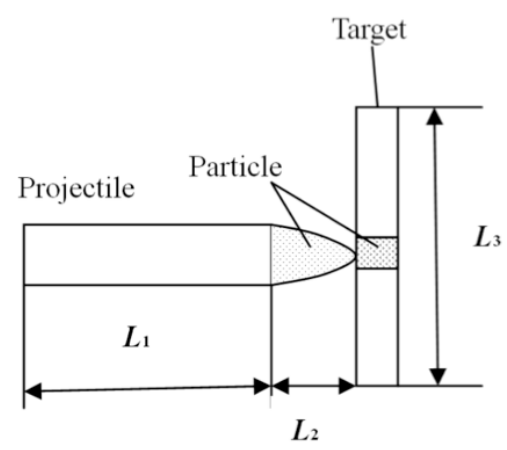

Fig. (4). The schematic representation of pointed projectile penetration into the steel target.

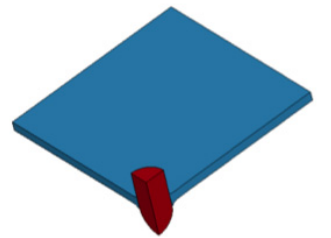

(a) $t=17.983 \mu \mathrm{s}$

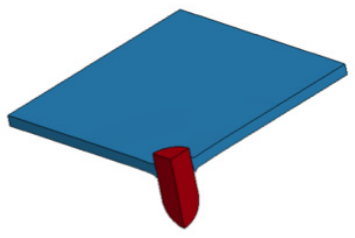

(b) $t=27.996 \mu \mathrm{s}$
Fig. (5). The penetration process at different time.

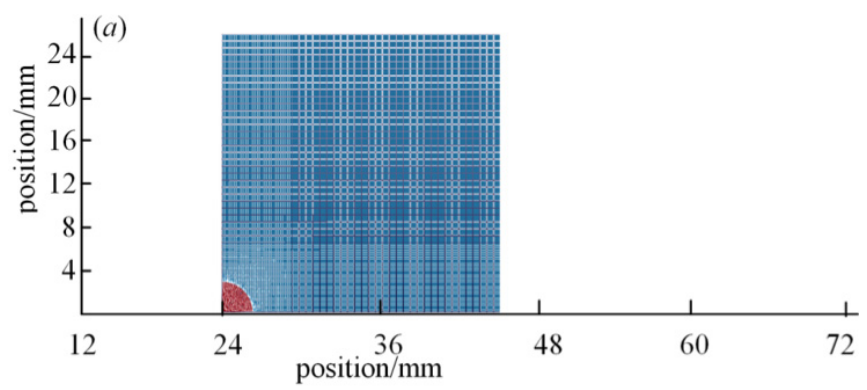

Fig. (6). The target plate deformation figure $(t=13.974 \mu \mathrm{s})$ (particle region). stress field and plastic strain field are shown in Fig. (7) and Fig. (8), respectively.

As can be seen from Fig. (5), the coupled level set and Particle-in-Cell method can track the deformation effectively in the process of penetration.

As can be seen from Fig. (7) and Fig. (8) projectile penetration is mainly extrusion damage based, and it shows in that the radius of the penetration channel increases gradually. At the same time, the material in front of the projectile are pushed to aside, and the petal-shaped are formed at the surface edges.

Fig. (9) shows the residual velocities from experimental and numerical simulations at different impact velocities. The computational results are in a good agreement with the experimental values, but higher than the experimental values in general. In addition, the lower the impact velocity is, the large errors of the residual velocities are. This is due to the hydro-elastic-plastic constitutive model used for the projectile and target materials. The higher the velocity is, the more similar is the material to the fluid state by the high temperature and high pressure.

\section{CONCLUSION}

The typical penetration problem such as pointed projectile penetration into steel target is numerically simulated by the coupled level set and Particle-in-Cell Method. The comparison of the numerical result and experimental result shows that the coupled algorithm can track the deformation effectively in the process of penetration and insures the precision and efficiency, and it can be extended to engineering design. 


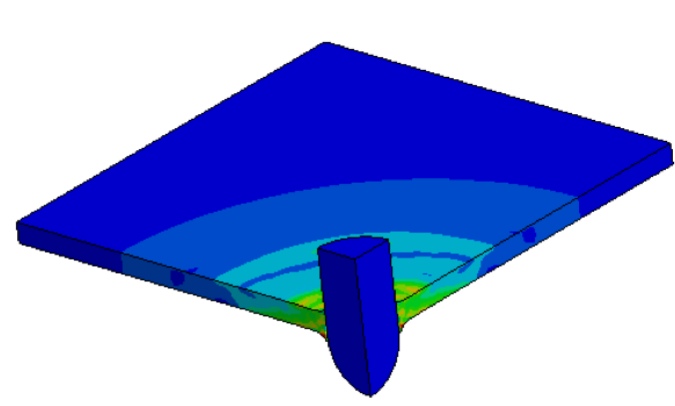

(a) $t=15.984 \mu \mathrm{s}$

Fig. (7). The stress field of the target.

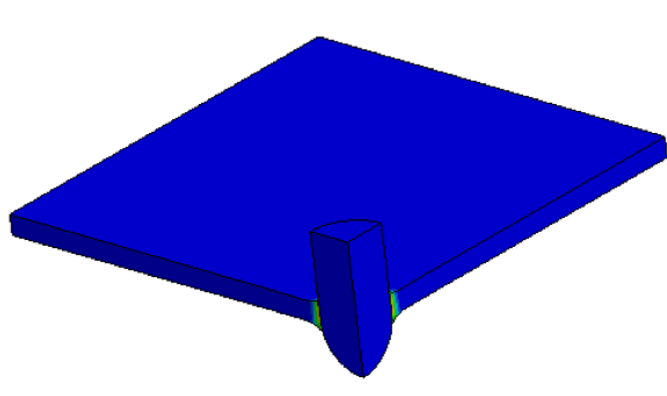

(a) $t=15.984 \mu \mathrm{s}$

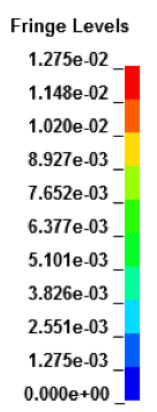

Fringe Levels

7.991e-01

$7.192 \mathrm{e}-01$

$6.393 \mathrm{e}-01$

$5.594 \mathrm{e}-01$

$4.795 \mathrm{e}-01$

$3.996 \mathrm{e}-01$

3.196e-01

2.397e- 01

$1.598 \mathrm{e}-01$

$7.991 \mathrm{e}-02$

$0.000 \mathrm{e}+00$

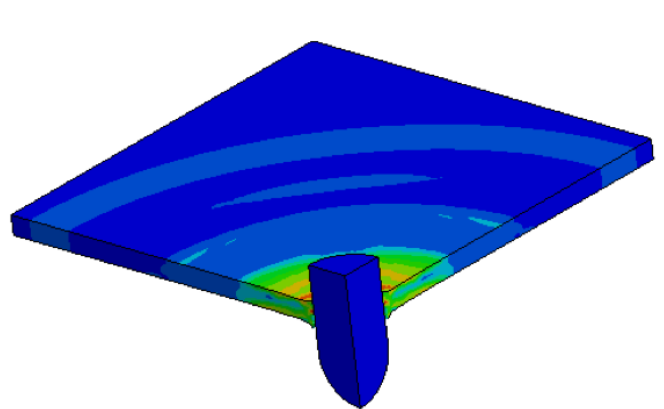

(b) $t=23.985 \mu \mathrm{s}$

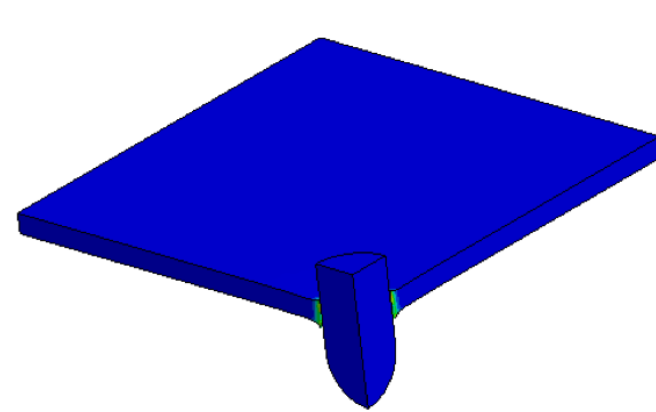

(b) $t=23.985 \mu \mathrm{s}$
Fringe Levels

1.232e-02

$1.109 \mathrm{e}-02$

$9.854 \mathrm{e}-03$

$8.622 \mathrm{e}-03$

$7.390 \mathrm{e}-03$

$6.159 \mathrm{e}-03$

$4.927 \mathrm{e}-03$

$3.695 \mathrm{e}-03$

$2.463 \mathrm{e}-03$

$1.232 \mathrm{e}-03$

$0.000 \mathrm{e}+00$

Fig. (8). The plastic strain field of the target.

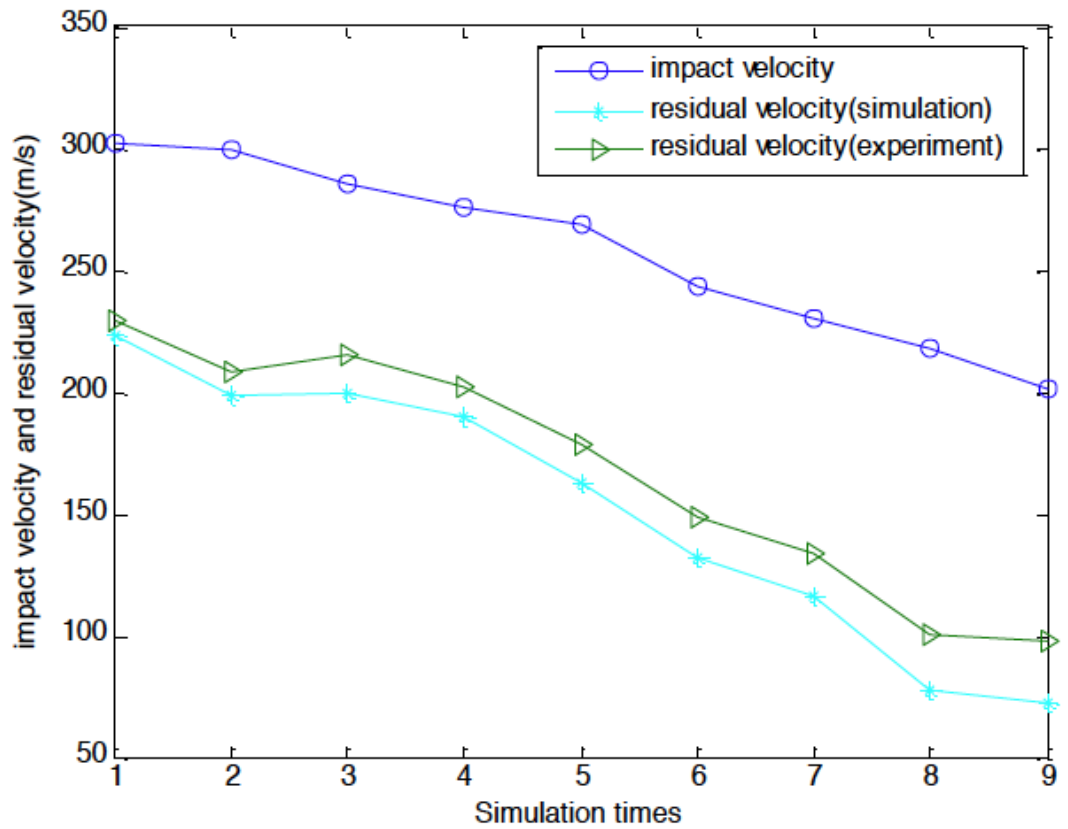

Fig. (9). The comparison of residual velocity and impact velocity.

\section{ABOUT THE AUTHORS}

First Author Zhang Li, master degree in Computational Physics and Engineering, studying for PhDs in Beijing Institute of Technology. The author's major is Computational Physics and Engineering. 7 papers received by EI or SCI.

Second Author Zhang Tao, Neijiang Normal University, instructor. The author's major is computational mathematics. 2 papers received by EI.
Third Author Wu Kaiteng, Neijiang Normal University, Ph.D. The author's major is Engineering Mechanics. 20 papers received by EI or SCI.

The last Author Ning Jianguo, Beijing Institute of Technology, Ph.D, Cheung Kong Scholars. The author's major is Explosive Mechanics. 80 papers received by EI or SCI. 


\section{CONFLICT OF INTEREST}

The authors confirm that this article content has no conflict of interest.

\section{ACKNOWLEDGEMENTS}

This work was financially supported by the education department of Sichuan Province project (12ZB266), the education department of Sichuan Province university innovation team (13TD0001), based on level set method of medical image three dimensional reconstruction algorithm research and its visualization (14CZ02).

\section{REFERENCES}

[1] J.F. Wang, "Penetration studies on the synthesis of the status quo", China Sci Technol Inf., vol. 6, pp. 129-130, 2005. (In Chinese).

[2] J.G. Ning, W.D. Song, and G.T. Yang, "Failure analysis of plastic spherical shell impacted by a projectile", Int. J. Impact. Eng., vol. 32, pp. 1464-1484, 2006.

[3] T.B. Ma, J. Wang, and J.G. Ning, "A hybrid VOF and PIC multimaterial interface treatment method and its aapplication in the penetration", Sci. China Phys. Mech. Astron., vol. 53, pp. 209-217, 2010.

[4] A.A. Amsden, "The particle in cell method for the calculations of the dynamics of compressible fluids", Los Alamos Scientific Laboratory Report LA, vol. 3466, 1996.

[5] F.H. Hardlow, and J.E. Welch, "Numerical calculation of time dependent viscous in compressible flow of fluid with free surface", Phys Fluids, vol. 8, pp. 21-82, 1965.

[6] R.A. Gebtry, R.E. Martin, and B.J. Daly, "An Eulerian differencing method for unsteady compressible flow problems", J. Comput.
Phys., vol. 1, pp. 87 -118, 1996.

[7] J.D. Kersher, and C.L. Mader, "A two-dimensional continuous Eulerian hydrodynamic code for computing multi-component reactive hydrodynamic problems", Los Alamos Scientific Laboratory Report LA, 4846, 1972.

[8] S.R. Yun, H.J. Tu, D.S. Liang, Computational method for explosion mechanics, CA, Beijing Institute of Technology Press, Beijing, pp. $201,1995$.

[9] S. Osher, and J.A. Sethian, "Fronts propagating with curvaturedependent speed: algorithms based on Hamiton-Jacobi formulations", J. Comput Phys., vol. 79, pp. 12-49, 1988.

[10] S. Osher, and R. Fedkiw, Level set methods and dynamic implicit surfaces, CA, Springer England, 2003, pp. 122-205.

[11] C.W. Hirt, and B.D. Nichols, "Volume of fluid (VOF) method for the dynamics of free boundaries", J. Comput. Phys., vol. 39, pp. 201-225, 1981.

[12] M. Sussmam, and E. Puckett, "An Adaptive Level-Set Approach for Incompressible Two Phase Flows", J. Comput Phys, vol. 148, pp. 81-124, 1999.

[13] D. Enright, R. Fedkiw, J. Ferziger, and I. Mitchell, "A hybrid particle level set method for improved interface capturing", J. Comput Phys, vol. 183. pp. 92-100, 2002.

[14] Y.C. Chang, Y.T. Hou, B. Merriman, and S. Osher, "A level-set Formulation of Eulerian Interface Capturing Methods for Incompressible Fluid Flows", J. Comput. Phys., vol. 124, pp. 449464, 1996.

[15] R.X. Liu, and Q.W. Shu, A number of new methods of computational fluid dynamics, Science Press Beijing, CA, 2003, pp. 101126.

[16] R. Fedkiw, A. Marquina, and B. Merriman, "An isobaric fix for the overheating problem in multimaterial compressible flows", J. Comput. Phys., vol. 148, pp. 545-578, 1999.

[17] K.T. Wu, L. Hao, C. Wang, and L. Zhang, "Euler method of Level Set interface processing and its application research", China scientific (G: physics), vol. 53, pp. 227-236, 2010.

Received: September 16,2014

Revised: December 23, 2014

Accepted: December 31, 2014

(C) Li et al.; Licensee Bentham Open.

This is an open access article licensed under the terms of the Creative Commons Attribution Non-Commercial License (http://creativecommons.org/licenses/by-nc/3.0/) which permits unrestricted, non-commercial use, distribution and reproduction in any medium, provided the work is properly cited. 